DOI 10.17816/transsyst201843s1289-297

(C) J.-W. Zeng ${ }^{1}$, Z.-Q. Long ${ }^{1}$, J. Ding ${ }^{1}$, X. Liang ${ }^{2}$

${ }^{1}$ National University of Defense Technology Maglev Engineering Research

${ }^{2}$ Hunan Maglev Technology Research Center Co., Ltd

(Changsha, China)

\title{
MEASUREMENT OF THE RESIDUAL STRESS FOR THE BOGIE FRAME OF MAGLEV VEHICLE BASED ON BARKHAUSEN EFFECT
}

Background: The effect of residual stress resulted from the manufacturing process on the performance of bogie frame has been attracting more and more attention with the increasing of running speed of maglev vehicle, which could develop cracks on the frame and compromise the operational safety.

Aim: It is necessary to determine and understand the distribution rules of residual stress on the bogie frames.

Methods: Barkhausen effect is one of the effective methods used to measure residual stress.

Results: This paper presents a experiment system designed for residual stress detection and the principle of electromagnetic stress detection is expounded, finite element simulation analysis on magnetization device is carried out, and the effect of magnetization under different excitation conditions is analyzed.

Conclusion: The feasibility of the online magnetic detection method of internal stresses in bogie frame is verified through the simulation, which would provided the basis for bogie frames internal stresses detection.

Keywords: bogie frames; maglev vehicle; Barkhausen effect; residual stress; nondestructive examination.

\section{INTRODUCTION}

The demand for train speed increase by users is increasing under the premise of the continuous development of medium-low speed maglev train. The operating environment of the maglev train is becoming more and more complicated when the train running speed is increased to $160-200 \mathrm{~km} / \mathrm{h}$. As the most critical part of the whole vehicle, the maglev train bogie supports the weight of the vehicle and transmits traction and braking force, which operating status is directly related to the safety of the entire vehicle. As the speed of the medium-speed maglev train increases, the traction force will increase appropriately on the basis of the original low-velocity traction force. The resulting stress and impact dynamic load 
of the bogie traction transmission will cause a surge of local stress, which result in increase in the stress and impact dynamic load of the bogie traction transmission causes a surge in local stress. Therefore, it is necessary to carry out non-destructive testing analysis of the internal stress distribution of the frame components and the service period of the materials.
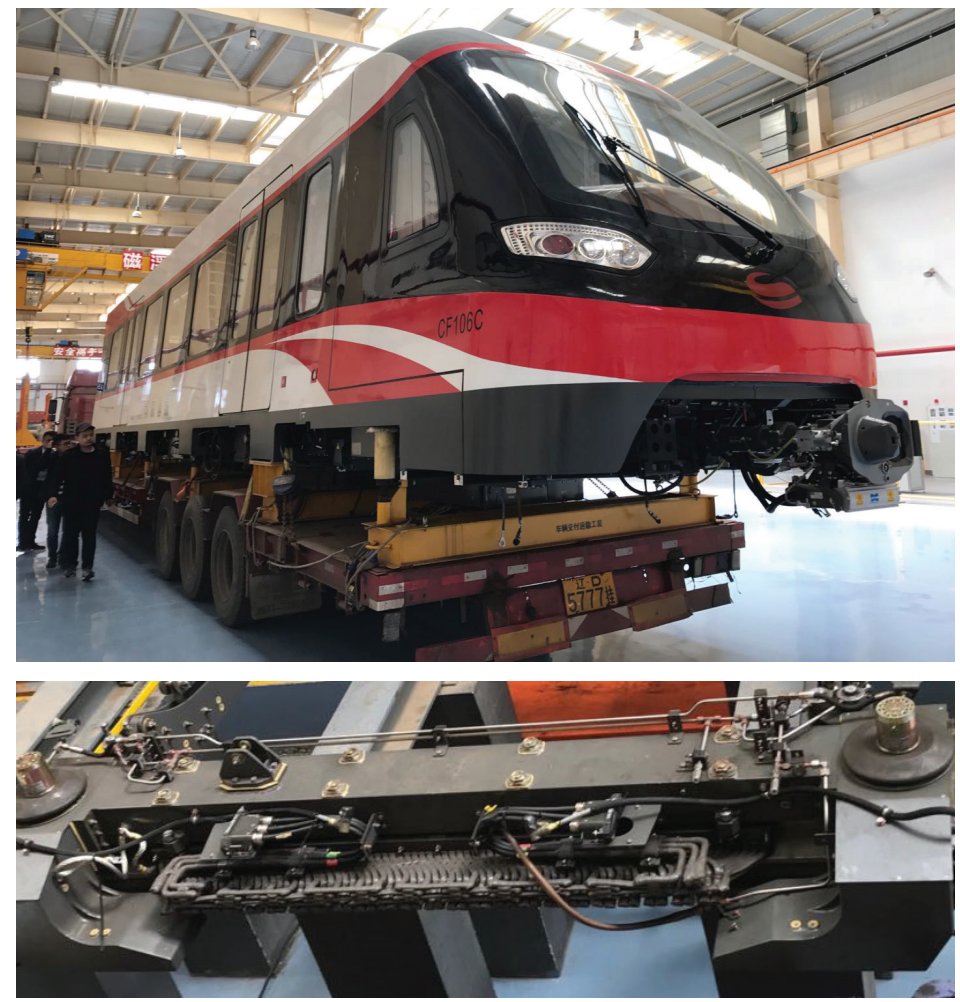

Fig. 1. Maglev train of Changsha Maglev Express

Non-destructive testing technology is playing an important role in the highlevel maintenance of urban rail vehicles. Nowadays the NDT methods mainly include magnetic powder, X-ray, ultrasonic and electromagnetic non-destructive testing. Among them, electromagnetic non-destruction has its unique advantages compared to other types of testing [1]. However, general electromagnetic nondestructive testing technology can't meet the current situation of steering frame maintenance. Magnetic Barkhausen noise on measurement of residual stresses in the nondestructive testing is a new technology [2]. In 1919, the German physicist Barkhausen the first time discovered the Barkhausen effect. He observed ferromagnetic hysteresis loop of materials and found: curve is not smooth, see changes which jitter level amplified, known as the Barkhausen transition. Nondestructive testing techniques based on the Barkhausen effect have little research on magnetic floatation [3-4]. Therefore, the development of the Barkhausen detection system for the bogie frame is particularly important. 
This paper presents a experiment system designed for residual stress detection and the principle of electromagnetic stress detection is expounded, finite element simulation analysis on magnetization device is carried out using ANSYS Maxwell, and the effect of magnetization under different excitation conditions is analyzed. The feasibility of the online magnetic detection method of internal stresses in bogie frame is verified through the simulation, which would provided the basis for bogie frames internal stresses detection.

\section{DETECTION PRINCIPLE}

The Barkhausen effect is a sudden discontinuous motion of the magnetic domain wall when the ferromagnetic material is magnetized to release elastic stress - strain wave phenomenon. The voltage pulse generated by this effect on the surface receiving coil of the sample is called Barkhausen noise, which is abbreviated as MBN [5].

According to the ferromagnetic theory, when the external magnetic field $\mathrm{H}=0$, the material is magnetically neutral and the domain walls are in equilibrium. When an external magnetic field is present, the domain wall displacement gradually increases from the reversible wall shifting stage. The strength required to move the domain wall to the demarcation point is the critical magnetic field strength $H_{0}$ :

$$
H_{0}=\frac{1}{2 \mu_{0} M_{s} \cos \theta}\left[\frac{d E}{d x}\right]_{\max }
$$

where $\mu_{0}$ is the magnetic permeability of material, $M_{s}$ is the saturation magnetization, $\theta$ is the small angle at which the magnetic domain moment is rotated by the action of the external magnetic field in the direction of easy magnetization, $E$ is the domain wall energy per unit area, $x$ is the displacement.

Wall shift occurs when the magnetic field strength increases slightly above $H_{0}$. As the strength of the magnetic field continues to increase, the domain wall again jumps, with $d E / d x$ further increasing. Therefore, as the strength of the magnetic field increases, several hopping domain wall movements may occur. All Barkhausen jumps in a complete magnetization process come together to form MBN.

During the magnetization of the material, microscopic defects and residual stresses inside the material can hinder the movement of the magnetic domain walls. In the process of magnetic domain wall displacement, it is necessary to overcome the potential energy barrier caused by uneven stress, impurities, holes and other factors inside the material. Therefore, the characteristics of the MBN signal can reflect the magnetic domain structure and motion law of the ferromagnetic material, which in turn reflects the microstructure and stress state of the material [6-8]. 
When the induction coil is placed on the surface of the material, it can receive the Barkhausen noise generated by the material during the magnetization process [9-10]. Through the acquisition and analysis of the noise signal, the relationship between the residual stress and other characteristics and the characteristic value of the Barkhausen noise signal is studied.

\section{TESTING TECHNIQUES AND INSTRUMENTS}

The functional blocks of the testing instruments are shown in Fig. 2. The excitation detecting sensor comprises a $U$ core magnet and a probe. The magnetization device is composed of a $\mathrm{U}$ core magnet and an exciting coil wound on the magnet. To produce Barkhausen noise, a magnetizing field is generated and applied to a ferromagnetic material through an electromagnet $[3,4]$. The material reacts to the magnetic field and emits Barkhausen noise, which are captured by a probe consisting of a coil of conducting wire. The signal is filtered and amplified. The amplitude is calculated and the data is digitized for display and output to storage meanwhile.

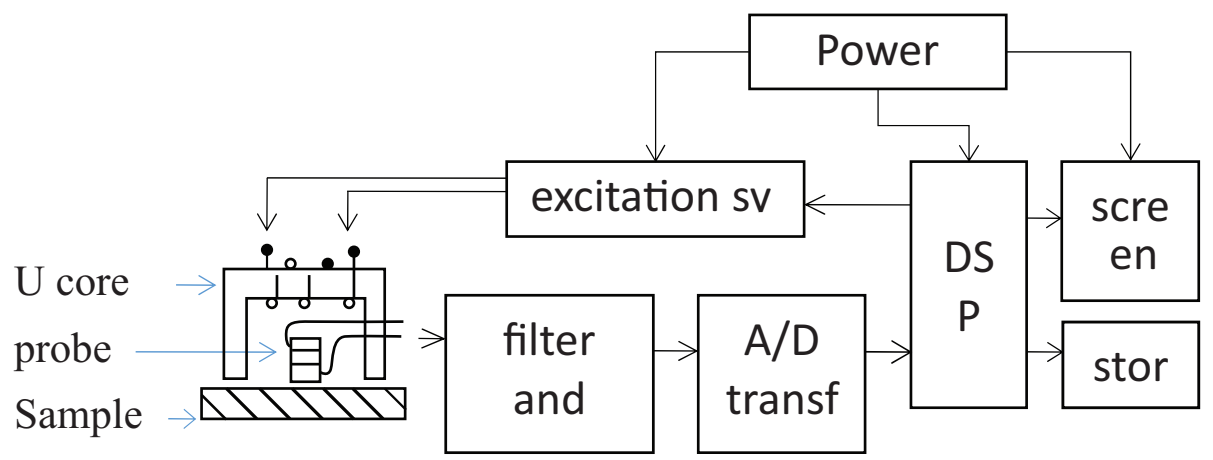

Fig. 2. The functional blocks of the testing instruments

\section{ESTABLISHMENT OF FINITE ELEMENT SIMULATION MODEL}

The simulation model is shown in Fig. 3. It mainly includes U-shaped magnetic core, excitation coil, and steel plate to be tested. Material properties assigned to the established electromagnetic mechanism: The magnetic core is made of cold rolled non-oriented silicon steel lamination, the material of the excitation coil is copper, the material to be tested is made of carbon steel, The geometric parameters of the excitation coil and specimen are listed in Tables 1, and 2. The boundary is set to the boundary of the balloon, and the inner and outer gaps of the sensor device are set to air. 


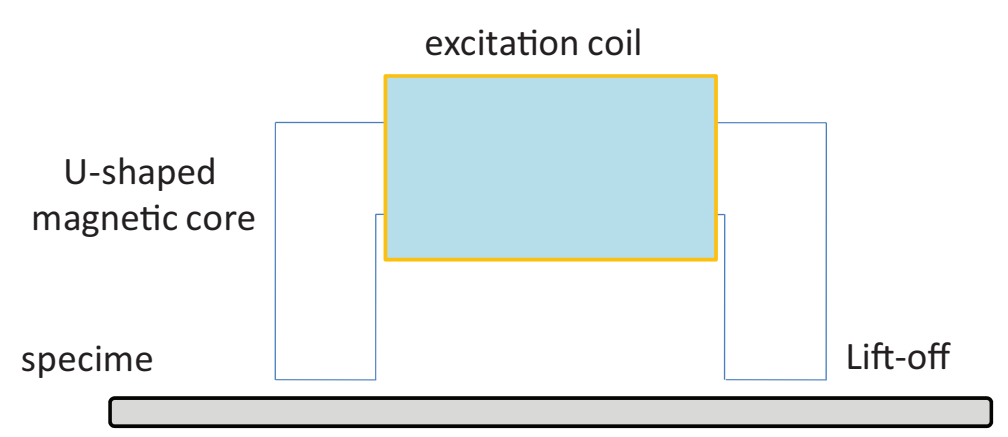

Fig. 3. Simulation model for excitation device

Table 1. Properties of the excitation coil

\begin{tabular}{|c|c|c|c|}
\hline Turns & $\begin{array}{c}\text { Cross-section } \\
\text { Shape }\end{array}$ & Material & $\begin{array}{c}\text { Current } \\
\text { source }\end{array}$ \\
\hline 300 & Rectangular & $\begin{array}{c}\text { Standard Copper } \\
\text { (Permeability }=1 \mathrm{Mu} ; \\
\text { Conductivity }=5.8 \mathrm{e} 007 \mathrm{~S} / \mathrm{m})\end{array}$ & $\begin{array}{c}1.0 \mathrm{~A} \\
\text { (Strand; Total) }\end{array}$ \\
\hline
\end{tabular}

Table 2. Dimension and properties of the conductive sample

\begin{tabular}{|c|c|c|c|}
\hline $\begin{array}{c}\text { Width } \\
(\mathbf{m m})\end{array}$ & $\begin{array}{c}\text { Thickness } \\
(\mathbf{m m})\end{array}$ & $\begin{array}{c}\text { Cross-section } \\
\text { Shape }\end{array}$ & Material \\
\hline 50 & 5 & Rectangular & $\begin{array}{c}\text { Iron (Permeability }=4000 ; \\
\text { having B-H curve) }\end{array}$ \\
\hline
\end{tabular}

Maxwell 3D software performs meshing methods automatically, adaptively and manually. The software recommends that the user manually split the data to more accurately analyze the static characteristics. Fig. 2 shows the result of manual splitting, defining the total number of cells of the core and the grid to be tested as 10000 . The mesh of the coil and air is adaptively split.

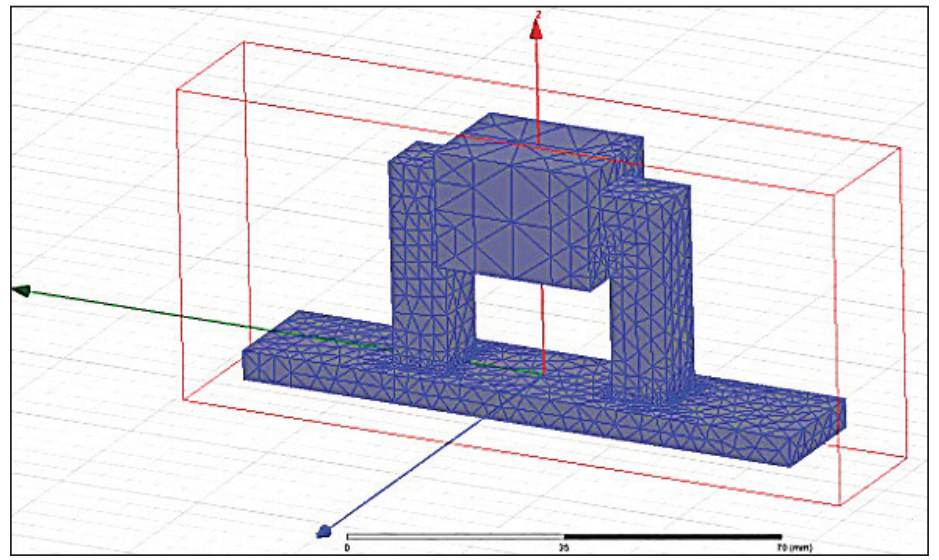

Fig. 4. Meshed model 


\section{NUMERICAL RESULTS}

\subsection{Magnetic Field Distribution}

The excitation coil is applied with a current of $1.0 \mathrm{~A}$, observe the distribution of the magnetic field lines in the magnetic permeability loop and the magnetic induction intensity distribution of the surface layer of the sample material. The magnetization effect is shown in Fig. 5 and Fig. 6, wherein Fig. 5 shows the magnetic field distribution of the magnetic permeability loop. Fig. 6 shows the magnetic induction distribution of the sample. It can be seen that the magnetic magnetic yoke and the sample material constitute a conduction magnetic circuit, and the surface layer portion of the sample reaches the magnetization effect. Therefore, the excitation device design meets the requirements, and the excitation effect on the test piece can be achieved.

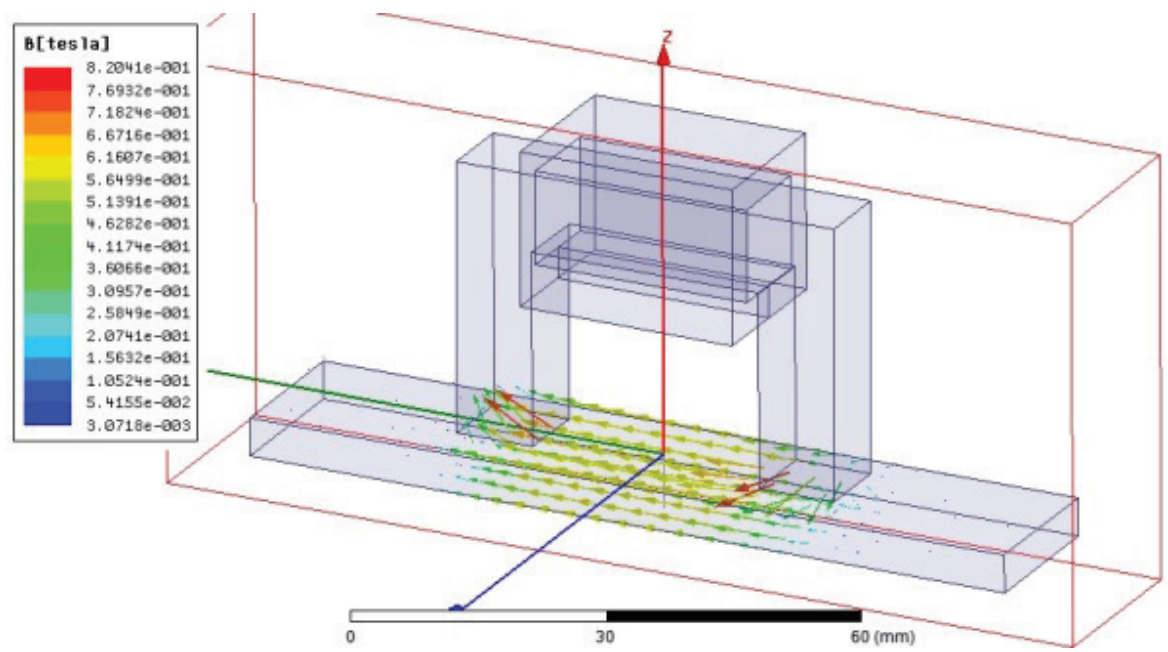

Fig. 5. B-vector map of steel plate
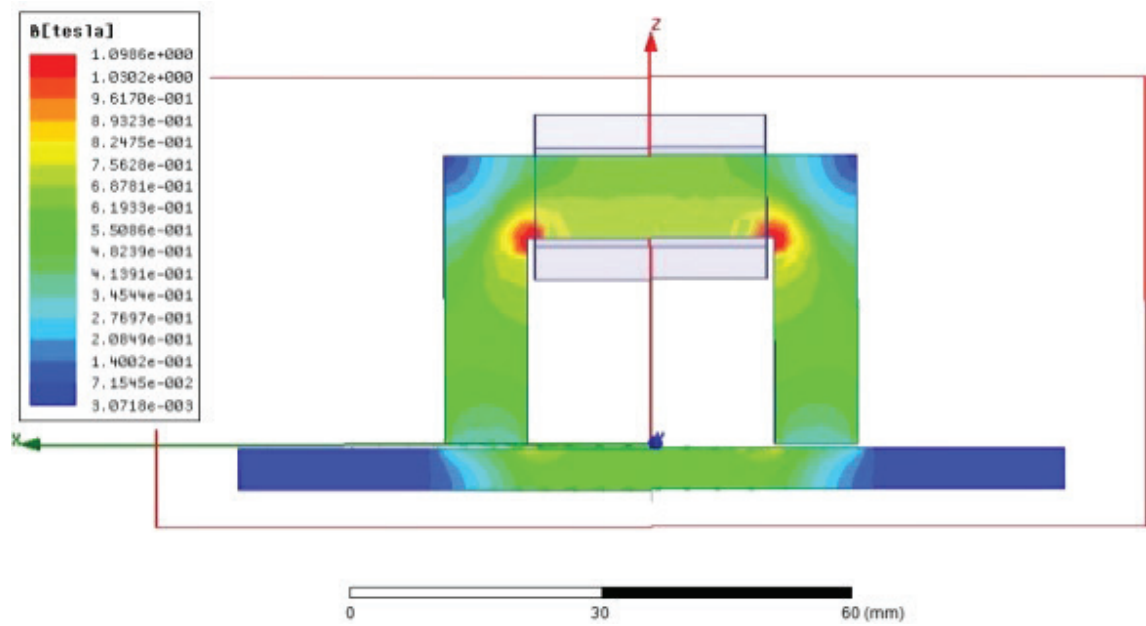

Fig. 6. Mag-B field map of xz plane 


\subsection{The Effect of Lift-off on Excitation}

Lift-off is a problem that has always been faced and needs to be solved in electromagnetic testing, and it has also become one of the main factors hindering the further development of electromagnetic defect detection technology. Liftoff refers to detecting the distance between the sensor and the tested part. When the relative position between the detecting sensor and the test piece changes, the degree of interaction between the detecting sensor and the test piece changes, and the detecting sensor and The effect of the change in distance between the test pieces on the detection of the sensor signal is called the "lift-off effect". In the Barkhausen excitation simulation experiment, and the aipgap is $0 \mathrm{~mm}-1.0 \mathrm{~mm}$, as shown in Fig. 7, the consequence of lift-off on the excitation effect. As the lift-off distance increases, the magnetic field induction intensity in the steel plate decreases exponentially, which is due to the increased distance between the detection sensor and the test piece, and the degree of interaction is weakened. Therefore, in order to improve the excitation effect of the Barkhausen effect excitation probe, the lift distance should be reduced as much as possible.

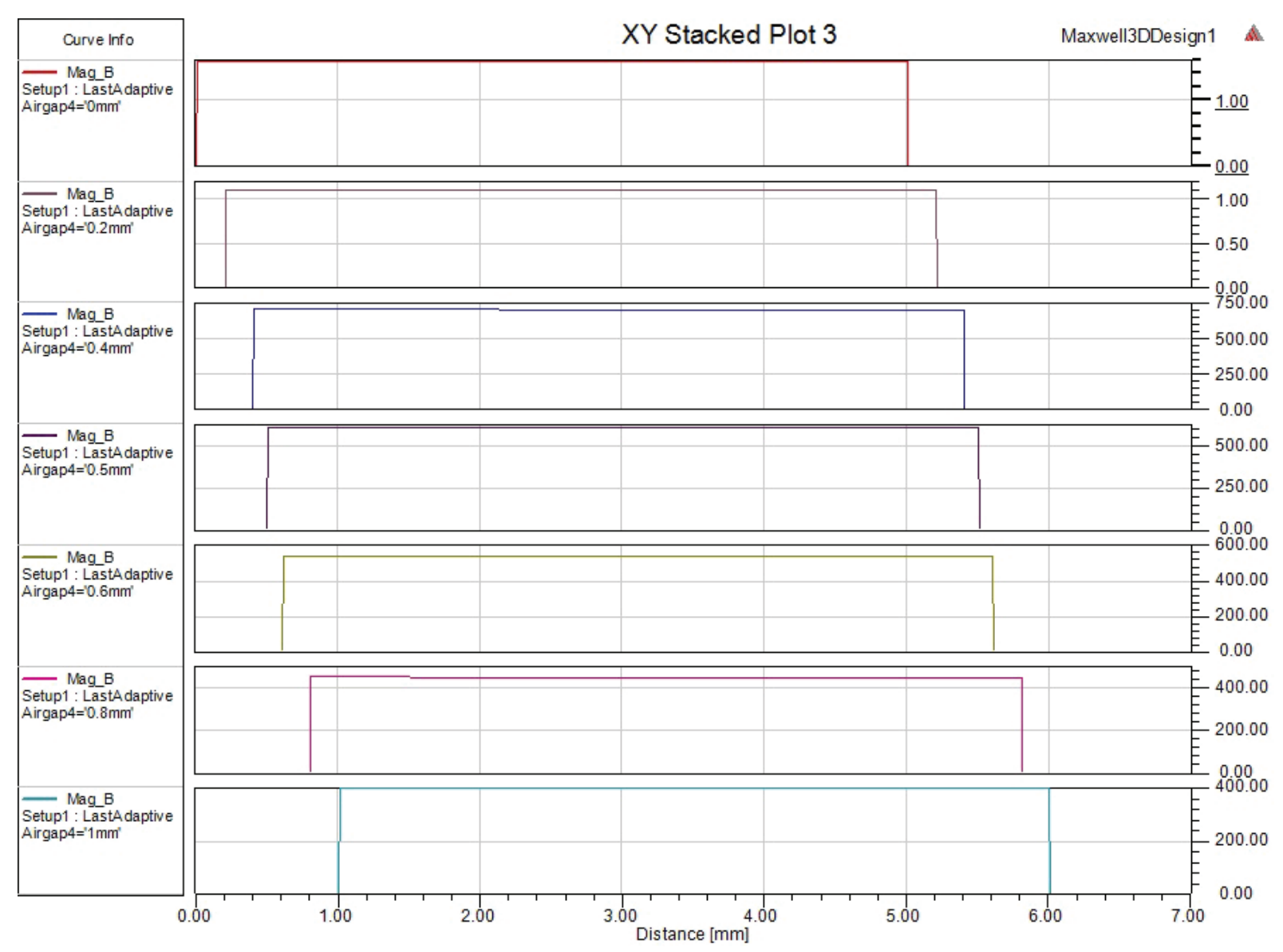

Fig. 7. Lift-off effects to sample for the airgap increased from $0 \mathrm{~mm}$ to $10 \mathrm{~mm}$ 


\section{CONCLUSION}

The feasibility of using the Barkhausen effect for detecting stress of bogie frame is investigated. According to the Barkhausen effect detection principle, an excitation detection sensor device is designed for the force-receiving parts of the medium-speed maglev train bogie frame. The electromagnetic field finite element analysis software is used to analyze the part of the designed magnetization device. From the above simulation analysis results, it can be concluded:

1) It can be seen from the magnetic line and the magnetic induction intensity map that the magnetic yoke and the material to be tested constitute a magnetic magnetic circuit, and the surface layer of the test piece achieves the magnetization effect. Therefore, the magnetizer meets the design requirements.

2) Lift-off is a key issue in electromagnetic testing. As the lift-off distance increases, the magnetic field induction intensity in the steel plate decreases exponentially. Therefore, in order to improve the excitation effect of the Barkhausen effect excitation probe, the lift distance should be reduced as much as possible.

The theoretical study demonstrates the feasibility of the proposed approach. Experimental validation of the inspection process is currently underway.

\section{ACKNOWLEDGMENT}

This paper is funded by National Key R\&D Program of China (No. 2016YFB1200602-19) and the Hunan Province Special Fund for the Strategic Emerging Industries and New Industrialization.

\section{References}

1. Kasai N, Koshino H, Sekine K, et al. Study on the Effect of Elastic Stress and Microstructure of Low Carbon Steels on Barkhausen Noise. Journal of Nondestructive Evaluation, 2013;32(3):277-285. doi: 10.1007/s10921-013-0180-1

2. Di Shu, Lei Guo, Liang Yin, et al. The effect of stress and incentive magnetic field on the average volume of magnetic Barkhausen jump in iron. Journal of Magnetism and Magnetic Materials, 2015;39(4):195-199. doi: 10.1016/j.jmmm.2015.06.059

3. Yang S, Sun Y, Udpa L, et al. 3D simulation of velocity induced fields for nondestructive evaluation application. IEEE Transactions on Magnetics, 1999;35(3): 1754-1756. doi: $10.1109 / 20.767369$

4. Tian GY, Li Y, Mandache C. Study of lift-off invariance for pulsed eddy-current signals, IEEE Trans. Magn. 2009;45(1):184-191. doi: 10.1109/tmag.2008.2006246

5. Yin Hechi, et al. Researching State and Development of Magnetic Barkhausen Noise in Measurement of Residual Stress. Nondestructive Testing, 2008;30(1):34-36. 
6. Li Y, Tian G Y, Ward S. Numerical simulations on electromagnetic NDT at high speed. Insight-Non-Destructive Testing and Condition Monitoring, 2006;48(2):103-108. doi: 10.1784/insi.2006.48.2.103

7. Li Y, Tian GY, Ward S. Numerical simulation on magnetic flux leakage evaluation at high speed, NDT \& E Int. 2006;39(5): 367-373. doi: 10.1016/j.ndteint.2005.10.006

8. Yin W, Hao XJ, Peyton AJ, Strangwood M, Davis CL. Measurement of decarburisation of steel rods with an electromagnetic sensor using an analytical model. NDT \& E Int. 2010;43(8):667-70. doi: 10.1016/j.ndteint.2010.07.003

9. Blaow M, Evans JT, Shaw BA. Surface decarburisation of steel detected by magnetic barkhausen emission. J Mater Sci 2004;40(20):5517-20. doi: 10.1007/s10853-005-4240-5

10. Mierczak LP, Melikhov Y, Jiles DC. Determining Residual Stress Depth Profiles Using the Magnetic Barkhausen Effect [J]. IEEE Transactions on Magnetics, 2014; 50(10):1-5. doi: 10.1109/tmag.2014.2329455

\section{Information about the authors:}

Zeng Jie-Wei, $\mathrm{PhD}$, Lecturer;

E-mail: yunsong118@163.com

Long Zhi-Qiang, PhD, Professor;

E-mail:631223920@qq.com

Ding Jingfang, $\mathrm{PhD}$, Professor;

E-mail: yunsong118@163.com

Liang Xiao, Engineer;

E-mail:2186075@qq.com

\section{To cite this article:}

Zeng J-W, Long Z-Q, Ding J, Liang X. Measurement of the Residual Stress for the Bogie Frame of maglev vehicle based on Barkhausen Effect. Transportation Systems and Technology. 2018;4(3 suppl. 1):289-297. doi: 10.17816/transsyst201843s 1289-297 